\title{
On the Kondo problem and thermodynamics of dilute magnetic alloys
}

\author{
Jan Maćkowiak* \\ Instytut Fizyki, Uniwersytet M. Kopernika, ul. Grudziadzka 5/7, 87-100 Toruń, Poland
}

(Dated: October 28, 2018)

\begin{abstract}
An argument is given showing that Coulomb attraction between conduction electrons and impurity ions in a dilute magnetic alloy (DMA) can be disregarded, provided the system's inverse temperature $\beta$ is replaced by an effective inverse temperature $t<\beta$. This replacement allows to remove the singularity in Kondo's expression for DMA impurity resistivity and extend his theory to $0 \mathrm{~K}$. The extended Kondo formula agrees with experimental data on resistivity of $\mathrm{CuFe}$ in the range of low temperatures and in the neighbourhood of the resistivity minimum.

Using an asymptotic solution of the thermodynamics of a dilute s-d system at inverse temperature $t$, the impurity thermodynamic functions are derived and shown to provide good agreement with experimental data on $\mathrm{CuFe}, \mathrm{CuCr}$ and $(\mathrm{LaCe}) \mathrm{Al}_{2}$ alloys in the low-temperature range. The magnitude of these functions agrees with experiment and does not require rescaling as in previous s-d theories. Nonlinear dependence of CuFe heat capacity on impurity concentration has been accounted for the first time.
\end{abstract}

PACS numbers: 72.15.Qm, 75.10.Jm, 75.30.Hx

Keywords: s-d exchange Hamiltonian; resistivity; heat capacity; magnetization; susceptibility

\section{INTRODUCTION}

The anomalous thermal behaviour of dilute magnetic alloys (DMA) has been the subject of extensive experimental and theoretical research over the past decades. The main stream of theoretical investigations (e.g. Refs. $1,2,3,3,4,5,5,6$, 7, 8, 10, 11) has focused on the construction of a conductivity theory and thermodynamics of DMA on the grounds of the s-d Hamiltonian $H_{\mathrm{s}-\mathrm{d}}$ introduced by Kasuya 212 .

Kondo's theory of DMA impurity resistivity $\Delta \rho$ 量 has successfully explained the experimentally observed dependence of $\Delta \rho$ on impurity concentration $c$ and temperature $T$ in the vicinity of the resistivity minimum. Unfortunately, the theory fails at $T=0$, where Kondo's expression for $\Delta \rho$ exhibits a logarithmic singularity. The question of removing this singularity is known as the "Kondo problem".

Substantial progress in understanding the anomalous DMA thermodynamics was made by Andrei et al.. .5 .6$ who solved $H_{\mathrm{s}-\mathrm{d}}$ thermodynamics for an s-d system with indistinguishable impurities and point s-d interaction. Their rescaled thermodynamic functions, agree, up to a small error, with experimental impurity specific heat and magnetization of $(\mathrm{LaCe}) \mathrm{Al}_{2}$. The solution found in Refs. 5 , 6 yields universal single-impurity curves for each thermodynamic function corresponding to a given value of impurity spin, which are independent of impurity concentration $c$.

The shape of experimental plots of DMA thermodynamic functions, in general, varies slowly with $c$ (e.g. Refs. 1, 13 14), meaning that their dependence on $c$ is nonlinear. This type of dependence has been accounted for by theory only in exceptional cases (e.g. Refs. 1, 15).

A different solution of $H_{\mathrm{s}-\mathrm{d}}$ thermodynamics, which treats a dilute s-d system (with a smeared s-d interaction) containing arbitrarily positioned distinguishable impurities and yields nonlinear dependence of thermodynamic functions on $c$, was presented by the author in Refs. 16, 17. The first quantization Hamiltonian $H_{\mathrm{s}-\mathrm{d}}$ in this approach is

$$
H_{\mathrm{s}-\mathrm{d}}^{(n, M)}=A^{(n)}\left(H_{0}^{(n)}+g^{2} \sum_{\alpha=1}^{M} \sum_{i=1}^{n} U\left(\mathbf{R}_{\alpha}-\mathbf{r}_{i}\right) \otimes S_{z \alpha} \sigma_{z i}\right),
$$

$n(M)$ denoting the number of electrons (impurities), $A^{(n)}$ the antisymmetrizer with respect to electron variables with indices $i=1, \ldots, n$, and

$$
H_{0}^{(n)}=-\sum_{i=1}^{n} \frac{\hbar^{2}}{2 m} \Delta_{i}
$$

$\mathbf{R}_{\alpha}$ denotes the position vector of the $\alpha$ th impurity, $S_{z \alpha}$ its spin operator and $\mathbf{r}_{i}, \sigma_{z i}$ stand for the respective quantities of the $i$ th electron. $U \geq 0$ represents any sufficiently regular function depending on $\left|\mathbf{R}_{\alpha}-\mathbf{r}_{i}\right|$, which allows application of the Feynman-Kac theorem to $\exp \left[-\beta H_{\mathrm{s}-\mathrm{d}}^{(n, M)}\right]^{18}$. This theorem was applied in Ref. 16 to derive an upper and lower bound to the system's free energy per electron,

$$
f\left(H_{\mathrm{s}-\mathrm{d}}^{(n, M)}, \beta\right):=-(n \beta)^{-1} \ln \operatorname{Tr} \exp \left[-\beta H_{\mathrm{s}-\mathrm{d}}^{(n, M)}\right],
$$

Typeset by REVTEX 
and to prove that the two bounds coaleesce in the limit of small $c \rightarrow 0$ (d-lim) and, as a consequence, that

$$
\lim _{n \rightarrow \infty} \mathrm{d}-\lim f\left(H_{\mathrm{s}-\mathrm{d}}^{(n, M)}, \beta\right)=\lim _{n \rightarrow \infty} \mathrm{d}-\lim \min _{\xi, \eta} f\left(h^{(n, M)}(\xi, \eta), \beta\right)=\lim _{n \rightarrow \infty} f\left(H_{0}^{(n)} A^{(n)}, \beta\right),
$$

where $h^{(n, M)}(\xi, \eta)$ is the mean-field Hamiltonian of an s-d system $S_{0}$ with separated electron and impurity variables. Both the electron subsystem $S_{\mathrm{e}}$ and the impurity subsystem $S_{\mathrm{imp}}$ of $S_{0}$ consist of noninteracting particles. According to Eq. (3),$h^{(n, M)}(\xi, \eta)$ is almost thermodynamically equivalent to $H_{\mathrm{s}-\mathrm{d}}$ in the extreme dilute limit.

The 1-electron Hamiltonian of $S_{\mathrm{e}}$ has the form

$$
h_{\mathrm{e}}^{(1, M)}(\xi, \eta)=\tilde{h}_{\mathrm{e}}^{(1, M)}(\xi, \eta)+\frac{1}{2} M\left(\xi^{2}-\eta^{2}\right) \mathbb{I}
$$

with

$$
\tilde{h}_{\mathrm{e}}^{(1, M)}(\xi, \eta)=H_{0}^{(1)}-g \sqrt{n}(\xi-\eta) \sum_{\alpha=1}^{M} U_{\alpha}^{(1)} \otimes \sigma_{z}^{(1)}
$$

$U_{\alpha}^{(1)}$ denoting the multiplication operator by $U\left(\mathbf{R}_{\alpha}-\mathbf{r}_{i}\right)$ and $\eta(\xi)=\xi-f_{2}(\xi)$, where

$$
f_{2}(\xi)=-\frac{g}{\sqrt{n}}\left\langle S_{z}\right\rangle_{h_{\mathrm{imp}}^{(1)}}, \quad\langle B\rangle_{h}:=\frac{\operatorname{Tr}(B \exp (-\beta h))}{\operatorname{Tr} \exp (-\beta h)},
$$

whereas $h_{\mathrm{imp}}^{(1)}$ is the 1-impurity Hamiltonian of $S_{\mathrm{imp}}$ :

$$
h_{\mathrm{imp}}^{(1)}(\xi)=g \sqrt{n} \xi S_{z \alpha}+\frac{1}{2} g^{2} S_{z \alpha}^{2} .
$$

The necessary condition for the minimum in Eq. (3) takes the form

$$
\xi=f_{3}(\xi)
$$

with

$$
\begin{gathered}
f_{3}(\xi):=f_{1}\left(f_{2}(\xi)\right)+f_{2}(\xi) \\
f_{1}(\xi):=g \sqrt{n}\left\langle\Gamma_{1}^{n} U_{\alpha}^{(1)} \sigma_{z}^{(1)}\right\rangle_{n \Gamma_{1}^{n} \tilde{h}_{\mathrm{e}}^{(1, M)}(\xi, 0)} \\
\Gamma_{1}^{n} B^{(1)}:=A^{(n)}\left(B^{(1)} \otimes \mathbb{I}^{(n-1)}\right) A^{(n)}
\end{gathered}
$$

$n \Gamma_{1}^{n} h^{(1)}$ denoting the Hamiltonian of $n$ noninteracting fermions with the 1-fermion Hamiltonian $h^{(1)}(\mathrm{cf}$. Ref. 19).

The mean-field thermodynamics founded on Eq. (3) was used in Ref. 17 to explain the presence of the impurity heat capacity peak of $\mathrm{CuCr}$ and $(\mathrm{LaCe}) \mathrm{Al}_{2}$ in the vicinity of the Kondo temperature $T_{K}$. In contradistinction to earlier papers (e.g. Refs. 5, 6, 20) scaling procedures were not used and nonlinear dependence of the CuCr peak's shape on $c$ was taken into account.

One of the shortcomings of DMA theories founded on s-d type Hamiltonians is the omission of the Coulomb attraction between impurity ions and conduction electrons. This problem has been treated in various ways in the past. Kondo introduced an additive term in his resistivity formula 1 to account for these interactions. In Refs. 21,22 the equilibrium state of an electron gas interacting with impurity ions was studied by averaging the 1-electron Green's function over impurity positions. By applying this method to the 1-particle equilibrium density matrix of a quantum particle in a field of randomly positioned wells, representing the screened Coulomb potential at each impurity site, it was shown in Ref. 17 that in the low temperature regime, a gas of such particles behaves effectively, with respect to 1-particle measurements, like a gas of free particles at an inverse temperature $t$ related to the system's real temperature $T$ by the equality

$$
t(\delta, T)=\delta^{-1} \tanh \left(\delta\left(k_{B} T\right)^{-1}\right)
$$


where $\delta=\frac{1}{2} \hbar \sqrt{M u_{2} m^{-1}}, u_{2}$ denoting the 2 nd derivative at the well's minimum. Accordingly, the inverse temperature $\beta=\left(k_{B} T\right)^{-1}$ of the s-d system under consideration will be subsequently replaced by $t(\delta, T+\Delta T)$ and $\delta, \Delta T$ will be treated as adjustable parameters. The shift $\Delta T$ is introduced in order to compensate omission of other DMA interactions in $H_{\mathrm{s}-\mathrm{d}}$.

In the high temperature range, $t(\delta, T)$ approaches smoothly $\beta$, whereas $\lim t(\delta, T)=\delta^{-1}$ as $T \rightarrow 0$. Replacement of $\beta$ by $t$ in Kondo's resistivity formula 1 , therefore allows to account for the Coulomb interactions between impurity ions and conduction electrons and to remove the singularity in his theory. In Section [II the resulting expression for DMA impurity resistivity $\Delta \rho$ is shown to give a good fit with experimental $\Delta \rho$ for CuFe with $c=110 \mathrm{ppm}$. Kondo's expression for the total DMA resistivity $\rho$ is also shown to comply with experimental $\rho$ under this substitution.

The objective of subsequent sections is to study the impurity heat capacity $\Delta C$, magnetization $\Delta M$ and susceptibility $\Delta \chi$ of the mean-field system $S_{0}$ and to adjust the constants which enter these thermodynamic functions to obtain the best possible agreement with experiment. For alloys with spin $1 / 2$ impurities, such as $\mathrm{CuFe}$ and $(\mathrm{LaCe}) \mathrm{Al}_{2}$, there is good agreement between theory and experiment, as regards dependence on $c$ and $T$ in the low-temperature range. In particular, nonlinear dependence of $\Delta C$ on iron concentration in CuFe has been accounted for successfully the first time. The magnitude of all thermodynamic functions agrees with experiment. For the $\mathrm{CuCr}$ alloys, containing spin $3 / 2$ ions, agreement is slightly weaker, presumably due to the simplicity of the assumed s-d interaction in Eq. (11), which permits only orbital s-wave scattering (cf. Ref. 2).

Computations were carried out using Wolfram's Mathematica 5.2.

\section{A SOLUTION OF THE KONDO PROBLEM}

In 1964 Kondo derived his well known formula 1 for the impurity resistivity of DMA:

$$
\Delta \rho=c \rho_{A}+c \rho_{M}\left(1-3 z g^{2} \varepsilon_{F}^{-1} \ln T\right)
$$

where $\rho_{A}, \rho_{M}, z$ are constants and $z$ is positive for antiferromagnetic s-d interaction. Inclusion of lattice resistivity $\rho_{L}=a T^{5}$ yields Kondo's expression for the total DMA resistivity $\rho$, which provides a good fit to the $c$, $T$ dependence of experimental data on resistivity of several DMA (cf. Ref. 1).

The breakdown of formula (13) at $T=0$ can be easily amended by noting that Kondo's theory takes into account the Coulomb attraction between conduction electrons and impurity ions simply by including an additive constant into the r.h.s. of Eq. (13). From the viewpoint of Eq. (12) it would be more appropriate to replace in Eq. (13) the true inverse temperature of the alloy by $t$. For $\Delta T=0$, the resulting expression for $\Delta \rho$ then takes the form

$$
\Delta \rho=\Delta \rho_{0}+\Delta \rho_{1} \ln \left(\tanh \left(\frac{\delta}{k_{B} T}\right)\right)
$$

and is regular at $T=0$.

Plausibility of formula (14) was tested by adjusting the constants $\Delta \rho_{0}, \Delta \rho_{1}, \delta$ to fit experimental $\Delta \rho(T)$ data for CuFe, with $c=22 \mathrm{ppm}$, plotted in Ref. 23. The function (14), for $\Delta \rho_{0}=1.455 / 10^{9} \mathrm{ohm} \mathrm{cm}$ per ppm, $\Delta \rho_{1}=$ $0.07 / 10^{9} \mathrm{ohm} \mathrm{cm}$ per ppm and $\delta=2 / 10^{4} \mathrm{eV}$, is depicted in Fig. 1. Agreement with experiment is good.

The experimental $\Delta \rho$ data for $\mathrm{CuFe}$ with $c=22 \mathrm{ppm}$ are quite typical. Resistivity measurements of a variety of DMA samples point to the close similarity of $\Delta \rho(T) / \Delta \rho(0)$ curves for various alloys 24 . Formula (14) can be therefore expected to provide good agreement with experiment for a large class of DMA.

Hamann's expression for impurity resistivity ${ }^{25}$, with $t$ replacing $\beta$, was also confronted with the data of Ref. 23. Qualitative agreement was found.

Performing the substitution $\beta \rightarrow t$ in Kondo's expression for total DMA resistivity $\rho$, one obtains

$$
\rho(T)=\Delta \rho(T)+\Delta \rho_{2}\left(\tanh \left(\frac{\delta}{k_{B} T}\right)\right)^{-5} .
$$

For $\Delta \rho_{0}=319.2937 \times 10^{-4} \mu \mathrm{ohm} \mathrm{cm}, \Delta \rho_{1}=2 \times 10^{-3} \mu \mathrm{ohm} \mathrm{cm}, \Delta \rho_{2}=1.065 \times 10^{-7} \mu \mathrm{ohm} \mathrm{cm}$ and $\delta=2.66 \times 10^{-4} \mathrm{eV}$, the function $\rho(T)$ provides a good fit to the experimental plot of $\rho(T)$ for CuFe with $c=1.23 \times 10^{-3}$ (Ref. 26) and is depicted in Fig. 2 .

The graphs of $k_{B} T$ and $t(\delta, T)^{-1}$ for $\delta=2.66 \times 10^{-4} \mathrm{eV}$ are plotted in Fig. 3. Close similarity of the two plots above $15 \mathrm{~K}$ shows that Eq. (15) provides an extension of Kondo's formula (14) to the vicinity of $0 \mathrm{~K}$. 


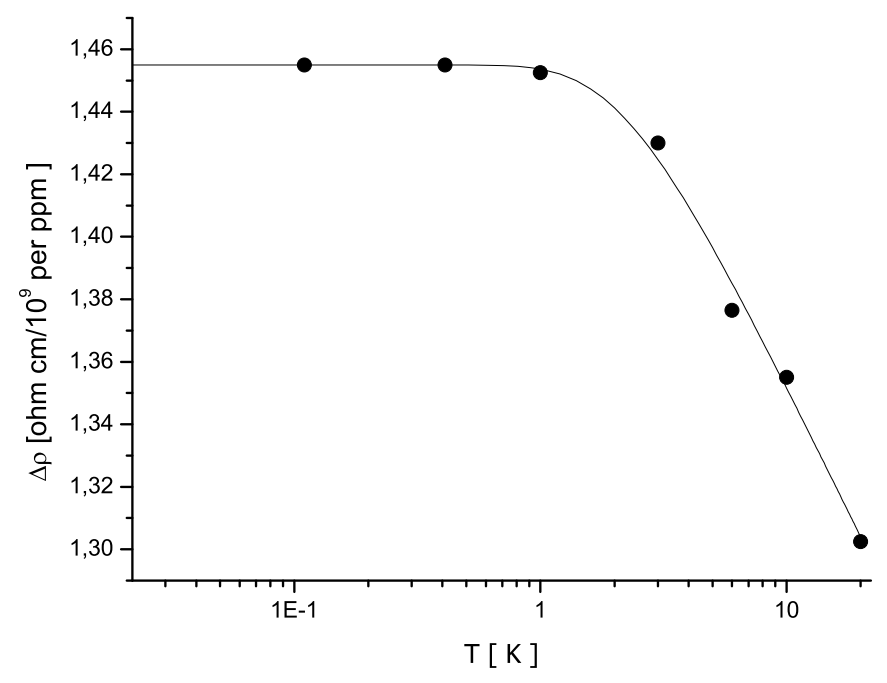

FIG. 1: Impurity resistivity $\Delta \rho$ of CuFe, with $c=22 \mathrm{ppm}$, as given by Eq. (14), for $\Delta \rho_{0}=1.455 / 10^{9}$ ohm per ppm, $\Delta \rho_{1}=$ $0.07 / 10^{9} \mathrm{ohm} \mathrm{cm}$ per ppm and $\delta=2 / 10^{4} \mathrm{eV}$. The points are experimental results from Ref. 23.

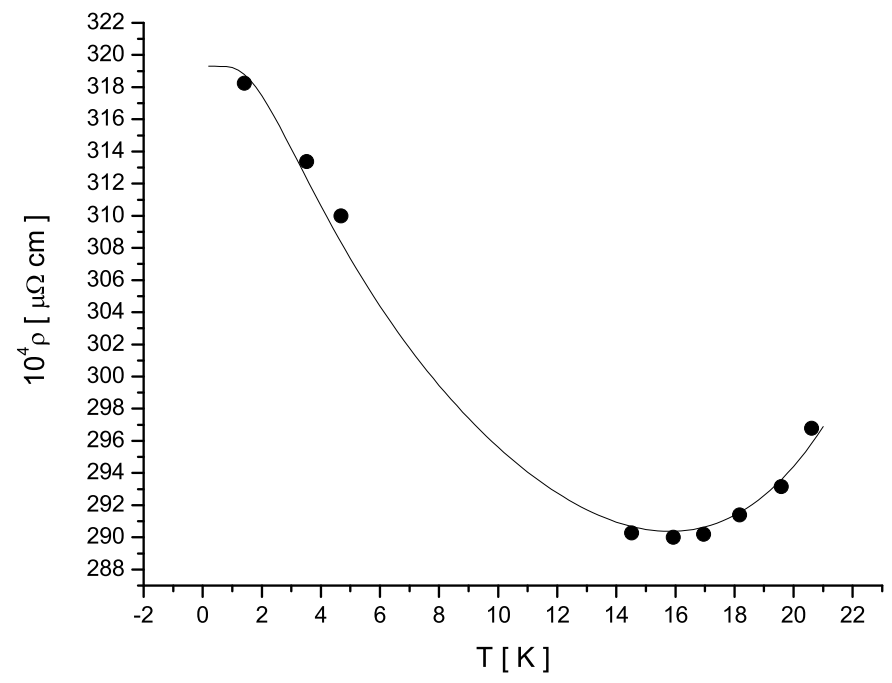

FIG. 2: The total resistivity $\rho$ of CuFe, with $c=1.23 / 10^{3}$, as given by Eq. 15), for $\Delta \rho_{0}=319.2937 / 10^{4} \mu \mathrm{ohm} \mathrm{cm}, \Delta \rho_{1}=$ $2 / 10^{3} \mu \mathrm{ohm} \mathrm{cm}, \Delta \rho_{2}=1.065 / 10^{7} \mu \mathrm{ohm} \mathrm{cm}$ and $\delta=2.66 / 10^{4} \mathrm{eV}$. The points are experimental results from Ref. 26 .

\section{MEAN-FIELD IMPURITY HEAT CAPACITY}

Expressions for the mean-field energy $\Delta U_{\mathrm{s}-\mathrm{d}}$ and heat capacity $\Delta C$ of a dilute s-d system, relative to that of a free electron gas, will be derived, using the Hamiltonian $h^{(n, M)}(\xi, \eta)$, and compared with experimental data on impurity heat capacity of $\mathrm{CuFe}^{13}, 27,(\mathrm{LaCe}) \mathrm{Al}_{2} \underline{28}$ and $\mathrm{CuCr}^{29}$. The system's inverse temperature $\beta$ will be replaced by $t(\delta, T)$. To compensate deficiencies of $H_{\mathrm{s}-\mathrm{d}}$, such as non-inclusion of the interaction between d-electrons present in the Anderson Hamiltonian, a shift $\Delta T>0$ in the temperature scale will be implemented (cf. Refs. 22, 28). Thus the effective inverse temperature (EIT)

$$
t(\delta, T+\Delta T)=\delta^{-1} \tanh \left(\delta\left(k_{B}(T+\Delta T)\right)^{-1}\right) .
$$




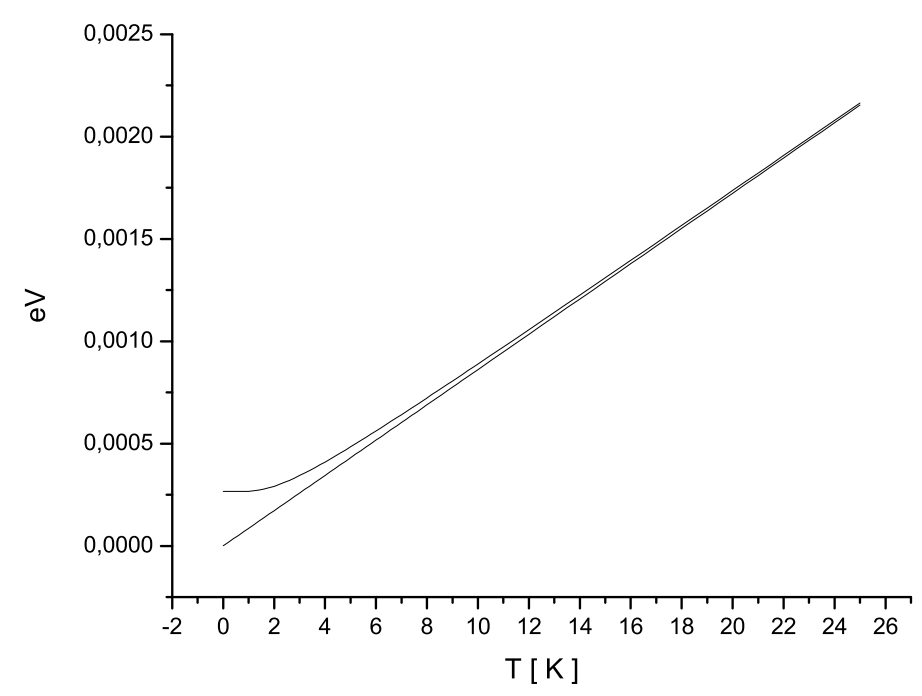

FIG. 3: The graphs of $k_{B} T$ and $t(\delta, T)^{-1}$ for $\delta=2.66 / 10^{4} \mathrm{eV}$.

\section{A. CuFe alloys}

The spin of the Fe ions in $\mathrm{CuFe}$ equals $1 / 2$ according to Ref. 27, therefore

$$
f_{2}(\xi)=\frac{\gamma}{M \sqrt{n_{1}}} \tanh \left(t(\delta, T+\Delta T) \gamma \sqrt{n_{1}} \xi\right)
$$

where $n=M n_{1}, \gamma=\sqrt{M} g, n_{1}$ denoting the number of conduction electrons per impurity. $f_{1}$ defined by Eq. (10) is a linear function in the simplest approximation: $f_{1}(\xi)=b_{0}+b_{1} \xi+\ldots \frac{17}{}$.

The expectation energy of a spin $1 / 2$ system $S_{\text {imp }}$ containing $M$ impurities with the 1-impurity Hamiltonian (7) equals

$$
U_{\mathrm{imp}}=\left\langle h_{\mathrm{imp}}^{(M)}\right\rangle_{h_{\mathrm{imp}}^{(M)}}=-M^{2} n_{1} \xi f_{2}(\xi)+\frac{1}{2} \gamma^{2}
$$

and, according to Appendix B of Ref. 17, the interaction energy of electrons with the Hamiltonian $\tilde{h}_{\mathrm{e}}^{(n, M)}(\xi, \eta)$ equals

$$
\Delta U_{\mathrm{e}}=\left\langle\tilde{h}_{\mathrm{e}}^{(n, M)}(\xi, \eta)\right\rangle_{\tilde{h}_{\mathrm{e}}^{(n, M)}(\xi, \eta)}-\left\langle n \Gamma_{1}^{n} H_{0}^{(1)}\right\rangle_{n \Gamma_{1}^{n} H_{0}^{(1)}}=-M n f_{2}(\xi)\left(b_{0}+b_{1} f_{2}(\xi)+\ldots\right),
$$

where $\xi$ is the minimizing solution of Eq. (8).

Using Eqs. (4), (18), (19) and the definition

$$
h^{(n, M)}(\xi, \eta):=h_{\mathrm{imp}}^{(M)}(\xi)+h_{\mathrm{e}}^{(n, M)}(\xi, \eta)
$$

one obtains

$$
\Delta U_{\mathrm{s}-\mathrm{d}}=U_{\mathrm{imp}}+\Delta U_{\mathrm{e}}+M n \xi f_{2}(\xi)-\frac{1}{2} M n f_{2}^{2}(\xi) .
$$

The $n$-electron, $M$-impurity spin $1 / 2 \mathrm{~s}$-d system will be now treated as a subsystem of a sample $S$ containing one mole of impurities. The energy $\Delta U_{\mathrm{S}}=6.022 \times 10^{23} M^{-1} \Delta U_{\mathrm{s}-\mathrm{d}}$ of such sample expressed in mcals, equals

$$
\Delta U_{\mathrm{S}}=602.2 \times 38271.78 M^{-1}\left(\frac{1}{2} \gamma^{2}-\frac{1}{2} M^{2} n_{1} f_{2}^{2}(\xi)-M^{2} n_{1} f_{2}(\xi)\left(b_{0}+b_{1} f_{2}(\xi)+\ldots\right)\right),
$$

if $b_{0}, \gamma, \delta, \xi$ are given in powers of $\mathrm{eV}$. 
TABLE I:

\begin{tabular}{c|c|c|c|c|c|c|c|c}
\hline \hline Alloy & $c$ & $n_{1}$ & $b_{0}\left[10^{-3} \sqrt{\mathrm{eV}}\right]$ & $b_{1}$ & $\gamma[\sqrt{\mathrm{eV}}]$ & $M$ & $\delta\left[10^{-4} \mathrm{eV}\right]$ & $\Delta T[\mathrm{~K}]$ \\
\hline $\mathrm{CuFe}$ & $0.05 \%$ & 2000 & 0.1 & -101 & 0.19 & $10^{8}$ & 7.14 & 2.6 \\
\hline $\mathrm{CuFe}$ & $0.1 \%$ & 1000 & 0.1 & -101 & 0.245 & $10^{9}$ & 7.48 & 3 \\
\hline $\mathrm{CuFe}$ & $0.2 \%$ & 500 & 0.1 & -101 & 0.288 & $10^{10}$ & 7.48 & 3.9 \\
\hline $\mathrm{CuCr}$ & $212 \times 10^{-7}$ & $10^{7} / 212$ & $1.01 / n_{1}$ & -631 & 0.086 & 36000 & $10^{-13}$ & 0.78 \\
\hline $\mathrm{CuCr}$ & $51 \times 10^{-6}$ & $10^{6} / 51$ & $1.09 / n_{1}$ & -461 & 0.091 & 248500 & $10^{-13}$ & 1.05 \\
\hline \hline
\end{tabular}

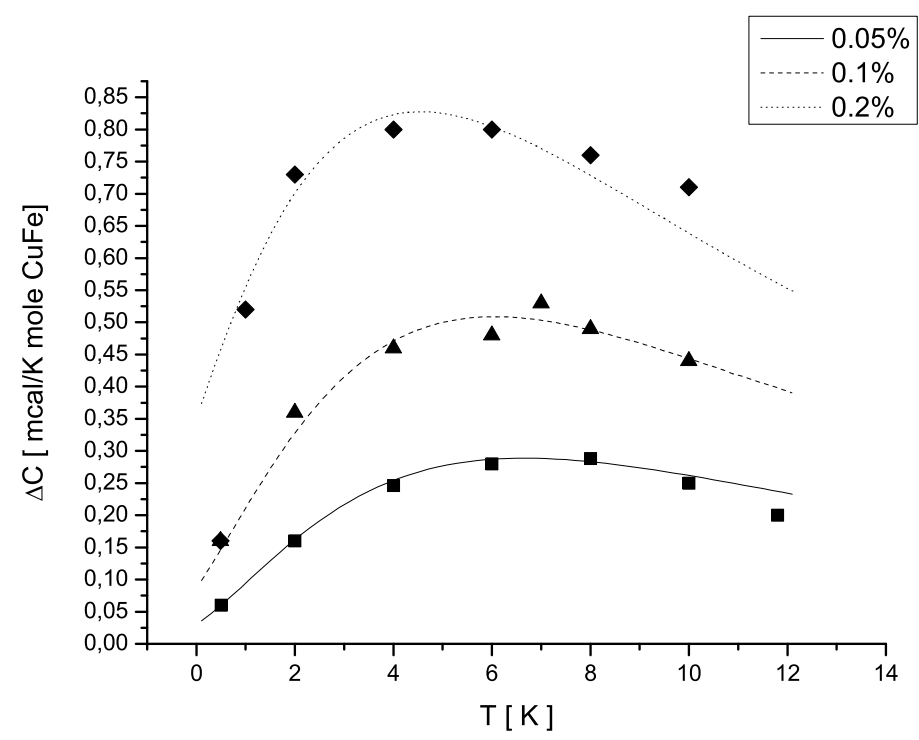

FIG. 4: Impurity heat capacity $\Delta C$ of CuFe given by Eq. (23), with $b_{0}, b_{1}, \gamma, M, \delta, \Delta T$ equal to the values in Table $\square$ The points are experimental results from Refs. 13 . 27.

The excess heat capacity of one mole of $\mathrm{CuFe}$ alloy, relative to that of one mole of pure $\mathrm{Cu}$, then equals

$$
\Delta C=n_{1}^{-1}\left(\frac{\partial \Delta U_{\mathrm{S}}}{\partial T}+\frac{\partial \Delta U_{\mathrm{S}}}{\partial \xi} \frac{\partial \xi}{\partial T}\right),
$$

where $\xi$ is the unique minimizing solution of Eq. (8) and

$$
\frac{\partial \xi}{\partial T}=-\frac{\partial f_{3}}{\partial T}\left(\frac{\partial f_{3}}{\partial \xi}-1\right)^{-1}
$$

The mean-field $\Delta C(T+\Delta T)$ curves best fitting to experimental data of Refs. 13, 27 were obtained for the values of $M, \gamma, b_{0}, b_{1}, \delta, \Delta T$ given in Table 1 and are depicted in Fig. 4. Agreement with experiment is good, especially for $c=0.05 \%, 0.1 \%$ and below $10 \mathrm{~K}$. Discrepancies at higher temperatures are presumably due to experimental error, which increases with temperature (e.g. Refs. 28, 29), and to an increase of the spin values of Fe ions in this temperature range $\frac{2,23}{2}$. In fact, Triplett et al ${ }^{29}$ estimate the spin of Fe ions in $\mathrm{CuFe}$ to be equal $3 / 2$.

The minimizing solutions of Eq. (8) are plotted in Fig. 5. Uniqueness of these solutions is proved in the Appendix.

It is worth emphasizing how significantly variation of $c$ affects the shape of both experimental and theoretical plots of $\Delta C(T+\Delta T)$ in Fig. 4 . It has been suggested by some authors (e.g. Refs. 29, 30) that nonlinearity of $\Delta C$ in $c$ observed in DMA is due to impurity-impurity interactions. The above analysis shows that this nonlinearity can be explained on the grounds of the s-d Hamiltonian without additional interaction terms.

Another remarkable property of the theoretical plots of $\Delta C(T+\Delta T)$ in Fig. 4 is their dependence on $M$. The best fitting values $M_{\mathrm{f}}$ of $M$ fall in the range $1 \ll M_{\mathrm{f}} \ll A=6.022 \times 10^{23}$. The sample $S$ can be therefore viewed as made up of magnetic domains, each containing $M_{\mathrm{f}}$ impurities with a favoured impurity-spin orientation, which varies, in general, 


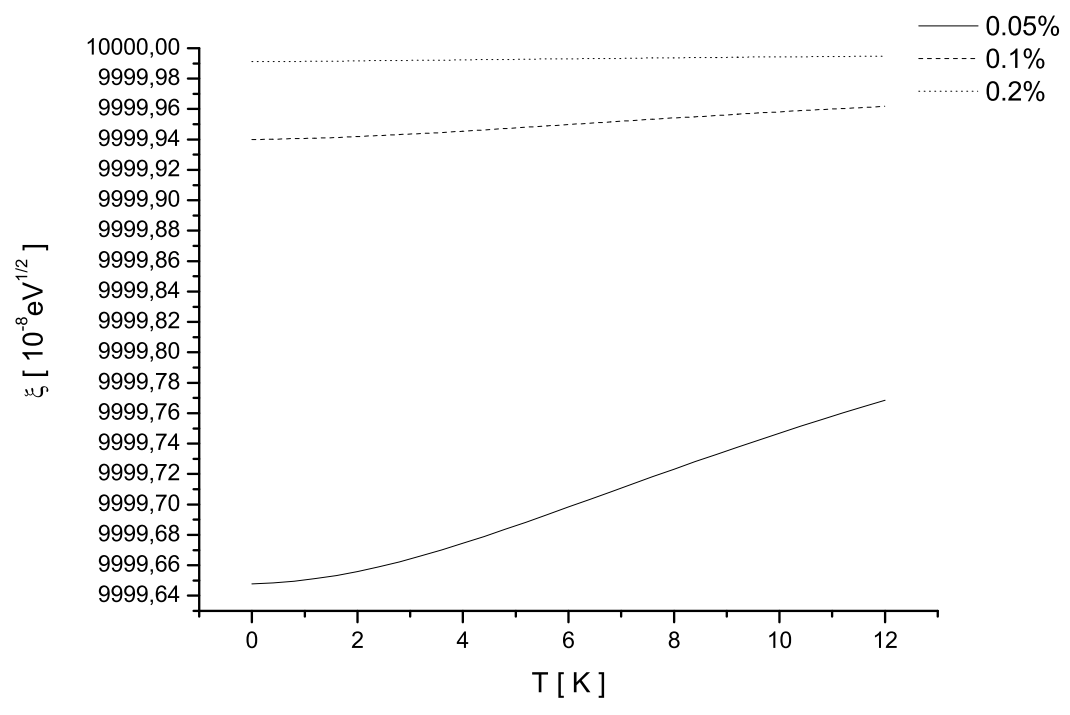

FIG. 5: The plots of the minimizing solutions $\xi(T+\Delta T)$ of Eq. (8) corresponding to the $\Delta C(T+\Delta T)$ curves of CuFe depicted in Fig. 4.

from one domain to another. Experiment has confirmed existence of magnetic domains in some magnetic materials (e.g. Ref. 31). The orientation of electron spins is opposite to that of impurities, as follows from Eqs. (5), (7).

Since the solution $\xi(T)$ of Eq. (8) decreases with decreasing $T$ (Fig. 51), it follows that the ordering of impurity spins declines as the temperature is lowered. A similar dependence of $\xi(T)$ on $c$ can be observed. In order to find lim $\xi(T)$ as $T \rightarrow 0$ for small enough $c$ let us recall that $\lim t(\delta, T)=\beta$ as $\delta \rightarrow 0$. The graphs of $\xi(T)$ for $t=\beta$, depicted in Fig. 3 of Ref. 17, as well as the form of Eq. (8) in all cases considered therein, show that as $T \rightarrow 0$, lim $\xi(T)=0$ if $\delta=0, \Delta T=0$. Hence, there is no ordering of impurity spins at $T=0$ if $\delta=0, \Delta T=0$, a picture which agrees with the RKKY description of interactions between impurity spins in a DMA.

\section{B. (LaCe) $\mathrm{Al}_{2}$}

Bader et al. ${ }^{28}$ performed interesting measurements of $\Delta C / c$ on $\left(\mathrm{La}_{1-x} \mathrm{Ce}_{x}\right) \mathrm{Al}_{2}$, with $x=0.0064$, in external magnetic fields ranging from $0 \mathrm{kOe}$ to $38 \mathrm{kOe}$. For strong fields Andrei et. al., obtained good agreement of their 1impurity $\Delta C(T)$ function with rescaled data of Ref. 28.

According to Refs. 14,28, a typical Kondo effect, without any superconducting side-effects observed in $\left(\mathrm{La}_{1-x} \mathrm{Ce}_{x}\right) \mathrm{Al}_{2}$ with Ce content exceeding $x=0.0067$. However, for $x=0.0064$, Bader et. al. estimate the difference between the expected normal state and measured superconducting-state heat capacities as insignificant. Thus a meanfield normal-state theory of $\Delta C / c$ for $\left(\mathrm{La}_{1-x} \mathrm{Ce}_{x}\right) \mathrm{Al}_{2}$, with $x=0.0064$, can be expected to provide good agreement with experiment.

The number of valence electrons per host atom in LaAl will be assumed 8/3. For $x=0.0064$,

$$
c=\frac{0.0064}{2.9936}=\frac{4}{1871}, \quad n_{1}=\frac{8}{3} c^{-1}=\frac{3742}{3} .
$$

The ground state of the Ce ion can be described by a spin with magnitude $1 / 2$ and a modified Lande factor $g^{\prime}=$ 10/7 (Ref. 14), therefore in the presence of an external magnetic field $H$, the system's Hamiltonian is

$$
H_{\mathrm{s}-\mathrm{d}}^{(n, M)}(H)=H_{\mathrm{s}-\mathrm{d}}^{(n, M)}-\frac{1}{2} g^{\prime} \mu_{B} \tilde{H} \sum_{\alpha} S_{z \alpha}-\frac{1}{2} \mu_{B} H A^{(n)} \sum_{i} \mathbb{I}_{0} \otimes \sigma_{z i},
$$

$\mathbb{I}_{0}$ denoting the identity in $L^{2}\left(\mathbb{R}^{3}\right)^{n}$ and

$$
f_{2}(\xi)=\frac{\gamma}{M \sqrt{n_{1}}} \tanh \left(t(\delta, T+\Delta T)\left(\gamma \sqrt{n_{1}} \xi-\frac{1}{2} g^{\prime} \mu_{B} \tilde{H}\right)\right),
$$




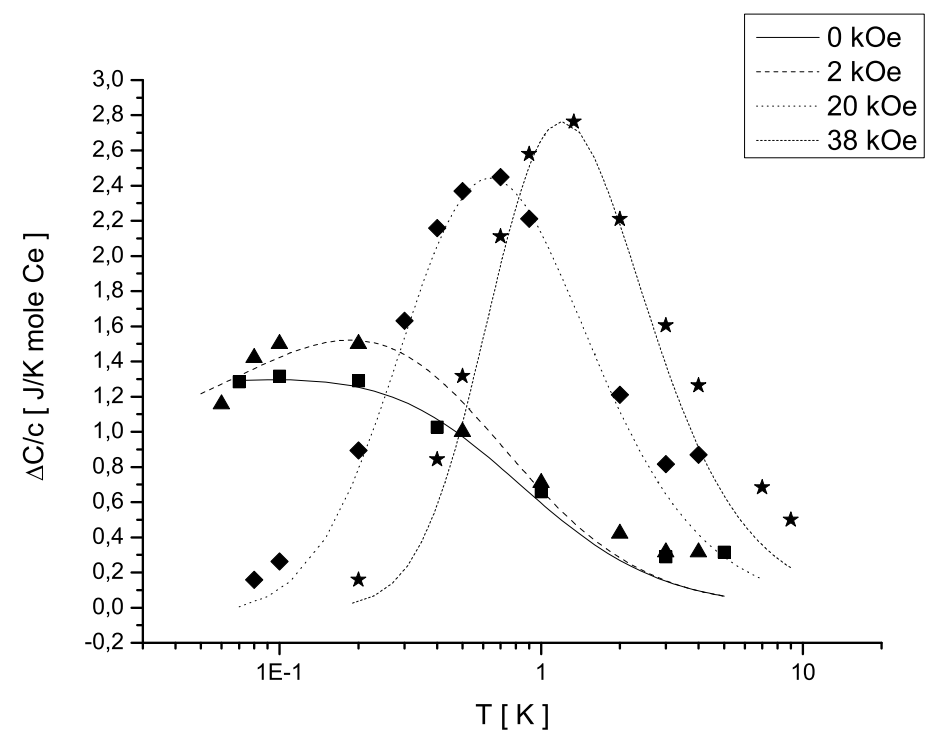

FIG. 6: The plots of $\Delta C(T+\Delta T) / c$ of $\left(\mathrm{La}_{1-x} \mathrm{Ce}_{x}\right) \mathrm{Al}_{2}$ in various external magnetic fields, as given by Eqs. (23), (27), for $x=0.0064$ and $b_{0}, b_{1}, \gamma, M, \delta, \Delta T, g_{0}$ equal to the values in Table $\amalg$. The points are experimental results from Ref. 28 .

TABLE II:

\begin{tabular}{c|c|c|r|c|c|c|c|c}
\hline \hline$b_{0}\left[10^{-4} \sqrt{\mathrm{eV}}\right]$ & $b_{1}$ & $\gamma\left[10^{-4} \sqrt{\mathrm{eV}}\right]$ & $M$ & $\delta\left[10^{-5} \mathrm{eV}\right]$ & $\Delta T[\mathrm{~K}]$ & $g_{0}$ & $H[\mathrm{kOe}]$ & $g^{2}\left[10^{-9} \mathrm{eV}\right]$ \\
\hline 1.9 & -13101 & 3.1 & 65 & 3.1215 & 0.39 & - & 0 & 1.4785 \\
\hline 1.9 & -13101 & 3.1 & 64 & 0.6243 & 0.27 & 0.006 & 2 & 1.50156 \\
\hline 1.9 & -13101 & 7.285 & 215 & 3.1215 & 0.11 & 0.006 & 20 & 2.46843 \\
\hline 1.9 & -13101 & 10.85 & 850 & 12.486 & 0.1 & 0.006 & 38 & 1.38497 \\
\hline \hline
\end{tabular}

where $\tilde{H}=g_{0} H$ is the effective magnetic field at each impurity site and $\mu_{B}$ the Bohr magneton. The excess energy $\Delta U_{S} / c$ of a sample $S$ of $(\mathrm{LaCe}) \mathrm{Al}_{2}$, with $x=0.0064$, expressed in joules, equals

$$
\begin{aligned}
c^{-1} \Delta U_{S} & =\frac{1}{4} M^{-1} 1871 \times 602.2 \times 160.2 \times \\
& \left(\frac{1}{2} \gamma^{2}-\frac{1}{2} M^{2} n_{1} f_{2}(\xi)^{2}-M^{2} n_{1} f_{2}(\xi)\left(\xi-f_{2}(\xi)\right)+\frac{1}{2} \gamma^{-1} \mu_{B} g^{\prime} \tilde{H} M^{2} \sqrt{n_{1}} f_{2}(\xi)\right)
\end{aligned}
$$

if $b_{0}, \gamma, \delta, \xi$ are expressed in powers of $\mathrm{eV}$. For one mole of impurities

$$
\Delta C=\frac{\partial \Delta U_{S}}{\partial T}+\frac{\partial \Delta U_{S}}{\partial \xi} \frac{\partial \xi}{\partial T}
$$

Adjusting the parameters $b_{0}, b_{1}, \gamma, M, \delta, \Delta T, g_{0}$, one obtains the best fitting to experiment $\Delta C(T+\Delta T) / c$ curves plotted in Fig. 6. The corresponding values of the parameters are given in Table II The mean-field thermodynamics founded on the Hamiltonian $h^{(n, M)}$ thus provides satisfactory agreement with experimental data on the field dependence of $\Delta C(T)$.

The value of $g_{0}$ was found by adjusting $\Delta C(T+\Delta T) / c$ to experiment for $H=2 \mathrm{kOe}$, with $b_{0}, b_{1}, \gamma$ equal to their best fitting values for $H=0$ and allowing only small variations of $M$. The smallness of $g_{0}$ indicates the strong influence of the Kondo effect in the formation of a polarization cloud of conduction electrons around each impurity. The cloud screens each magnetic ion from interactions with other magnetic ions ${ }^{27}$ and, as implied by the inequality $g_{0} \ll 1$, also from the applied field $H$. 


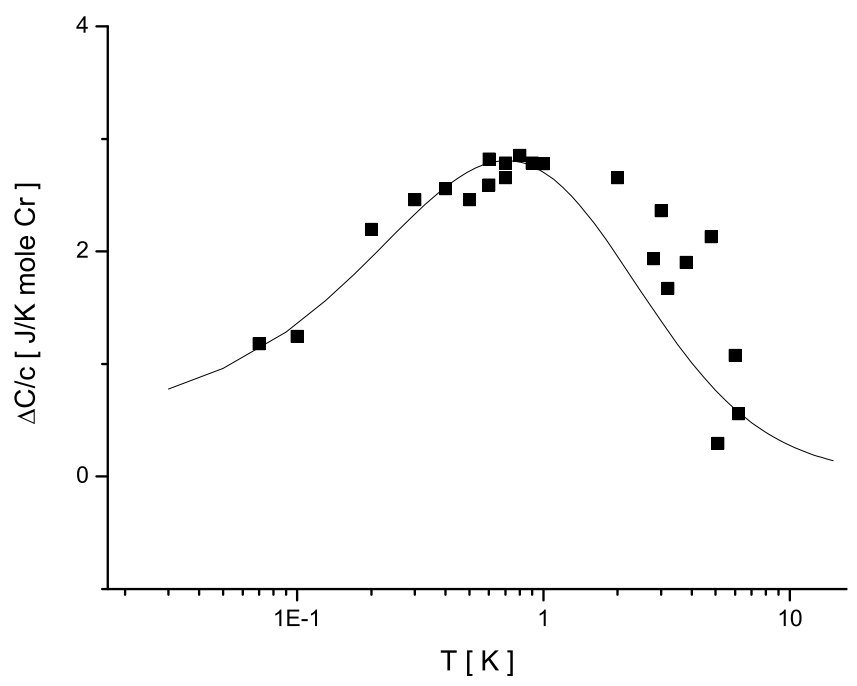

FIG. 7: The graph of $\Delta C(T+\Delta T) / c$ of $\mathrm{CuCr}$ with $c=21.7 \mathrm{ppm}$ as given by Eqs. (23), (31), with $b_{0}, b_{1}, \gamma, M, \delta, \Delta T$ equal to the values in Table I The points are experimental results from Ref. 29.

\section{CuCr alloys}

Triplett et. al $\stackrel{29}{\underline{ }}$ performed high-precision measurements of $\mathrm{CuCr}$ impurity heat capacity $\Delta C$ for a variety of concentrations. For $c=51 \mathrm{ppm}$ their $\Delta C(T)$ peak is well defined and terminates at low temperatures with a $\Delta C$ jump which they consider to be the effect of impurity-impurity interactions. Explanation of these experimental $\Delta C(T)$ data above the jump temperature is a challenge to any s-d theory.

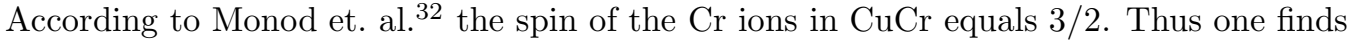

$$
f_{2}(\xi)=\frac{\gamma}{M \sqrt{n_{1}}} \frac{3 \mathrm{e}^{-4 t \gamma^{2} M^{-1}} \sinh \left(3 t \gamma \sqrt{n_{1}} \xi\right)+\sinh \left(t \gamma \sqrt{n_{1}} \xi\right)}{\mathrm{e}^{-4 t \gamma^{2} M^{-1}} \cosh \left(3 t \gamma \sqrt{n_{1}} \xi\right)+\cosh \left(t \gamma \sqrt{n_{1}} \xi\right)}
$$

and

$$
U_{\mathrm{imp}}=\left\langle h_{\mathrm{imp}}^{(M)}\right\rangle_{h_{\mathrm{imp}}^{(M)}}=-M^{2} n_{1} \xi f_{2}(\xi)+\frac{1}{2} \gamma^{2}+f_{4}(\xi)
$$

where

$$
f_{4}(\xi)=4 \gamma^{2} \frac{\mathrm{e}^{-4 t \gamma^{2} M^{-1}} \cosh \left(3 t \gamma \sqrt{n_{1}} \xi\right)}{\mathrm{e}^{-4 t \gamma^{2} M^{-1}} \cosh \left(3 t \gamma \sqrt{n_{1}} \xi\right)+\cosh \left(t \gamma \sqrt{n_{1}} \xi\right)} .
$$

Using Eqs. (19), (21), one obtains the following formula for $\Delta U_{S}$ (expressed in joules) of a sample $S$ of $\mathrm{CuCr}$ :

$$
\Delta U_{S}=602.2 \times 160.2 \times M^{-1}\left(\frac{1}{2} \gamma^{2}+f_{4}(\xi)-\frac{1}{2} M^{2} n_{1} f_{2}^{2}(\xi)-M^{2} n_{1} f_{2}(\xi)\left(\xi-f_{2}(\xi)\right)\right),
$$

if $b_{0}, \gamma, \delta, \xi$ are given in powers of eV. $\Delta C(T+\Delta T)$ obtains using Eqs. (24), (28).

The best fitting graphs of $\Delta C(T+\Delta T) / c$ for $c=21.7 \mathrm{ppm}$ and $c=51 \mathrm{ppm}$ are plotted in Figs. 7, 8, The corresponding values of parameters $b_{0}, b_{1}, \gamma, M, \delta, \Delta T$ are given in Table $\square$.

Agreement with experimental data of Ref. 29 is satisfactory, especially for $c=21.7 \mathrm{ppm}$, although not as good as for $\mathrm{CuFe}$ (Section IIIA), presumably due to simplicity of the assumed s-d interaction in Eq. (11) and variation of $\mathrm{Cr}$ spin values at higher temperatures. It has been suggested ${ }^{2.33}$ that for larger impurity spins the s-d interaction should account for the momentum dependence of s-d coupling. 


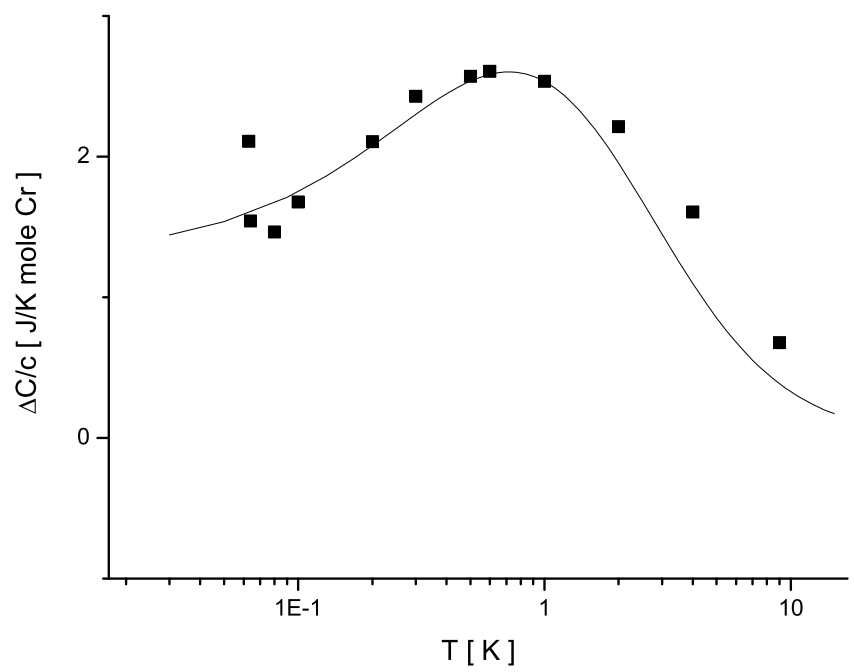

FIG. 8: The graph of $\Delta C(T+\Delta T) / c$ of $\mathrm{CuCr}$ with $c=51 \mathrm{ppm}$ as given by Eqs. (23), (31), with $b_{0}, b_{1}, \gamma, M, \delta, \Delta T$ equal to the values in Table【 The points are experimental results from Ref. 29.

TABLE III:

\begin{tabular}{|c|c|c|c|c|c|c|c|c|}
\hline$b_{0}\left[10^{-11} \sqrt{\mathrm{erg}}\right]$ & $b_{1}$ & $\gamma\left[10^{-10} \sqrt{\mathrm{erg}}\right]$ & $M$ & $\delta\left[10^{-17} \mathrm{erg}\right]$ & $\Delta T[\mathrm{~K}]$ & $g_{0}$ & $H[\mathrm{kOe}]$ & $g^{2}\left[10^{-24} \mathrm{erg}\right]$ \\
\hline 28.8576 & -13101 & 3.9237 & $10^{4}$ & 2 & 0.045 & 0.006 & 1 & 15.3954 \\
\hline 38.4768 & -13101 & 7.8474 & $2 \times 10^{4}$ & 3.1 & 0.094 & 0.006 & 2 & 30.7908 \\
\hline 48.096 & -13101 & 19.6185 & $2.5 \times 10^{4}$ & 4 & 0.18 & 0.006 & 5 & 153.9542 \\
\hline 48.096 & -13101 & 39.237 & $3 \times 10^{4}$ & 4.2 & 0.23 & 0.006 & 10 & 513.1807 \\
\hline
\end{tabular}

\section{MAGNETIZATION OF (LaCe) $\mathrm{Al}_{2}$}

Various measurements of DMA impurity magnetization $\Delta M$ (e.g. Ref. 14 and references therein) point to a similar field dependence of the $\Delta M(H, T)$ vs. $H / T$ curves. A typical experimental plot of $\Delta M(H, T)$, for $\left(\mathrm{La}_{1-x} \mathrm{Ce}_{x}\right) \mathrm{Al}_{2}$ with $x=0.015$, can be found in Ref. 14 . The single-impurity theoretical $\Delta M(H, T)$ curves found by Andrei et al ${ }^{5,6}$ fit the rescaled data of Ref. 14 up to a small error.

As implied by the form of the mean-field counterpart of the Hamiltonian (25), viz.,

$$
h^{(n, M)}(H)=h^{(n, M)}(\xi, \eta)-\frac{1}{2} g^{\prime} \mu_{B} \tilde{H} \sum_{\alpha} S_{z \alpha}-\frac{1}{2} \mu_{B} H \sum_{i} \mathbb{I}_{0} \otimes \sigma_{z i} A^{(n)},
$$

for a mole of spin $1 / 2$ impurities

$$
\Delta M=\frac{1}{2} g^{\prime} \mu_{B} \sum_{\alpha=1}^{A}\left\langle S_{z \alpha}\right\rangle_{h_{\mathrm{imp}}^{(A)}}=\frac{1}{2} g^{\prime} \mu_{B} A \tanh \left(t(\delta, T+\Delta T)\left(\frac{1}{2} g^{\prime} \mu_{B} \tilde{H}-\gamma \sqrt{n_{1}} \xi\right)\right)
$$

where $\xi$ is the unique solution of Eq. (8) with $f_{2}(\xi)$ given by Eq. (26). The resulting plots of $\Delta M(H, T)$ for various applied fields are depicted in Fig. 9. The corresponding values of $b_{0}, b_{1}, \gamma, M, \delta, g_{0}$ are presented in Table III. Agreement with experiment is good in the range of low temperatures, but less satisfactory at higher $T$. 


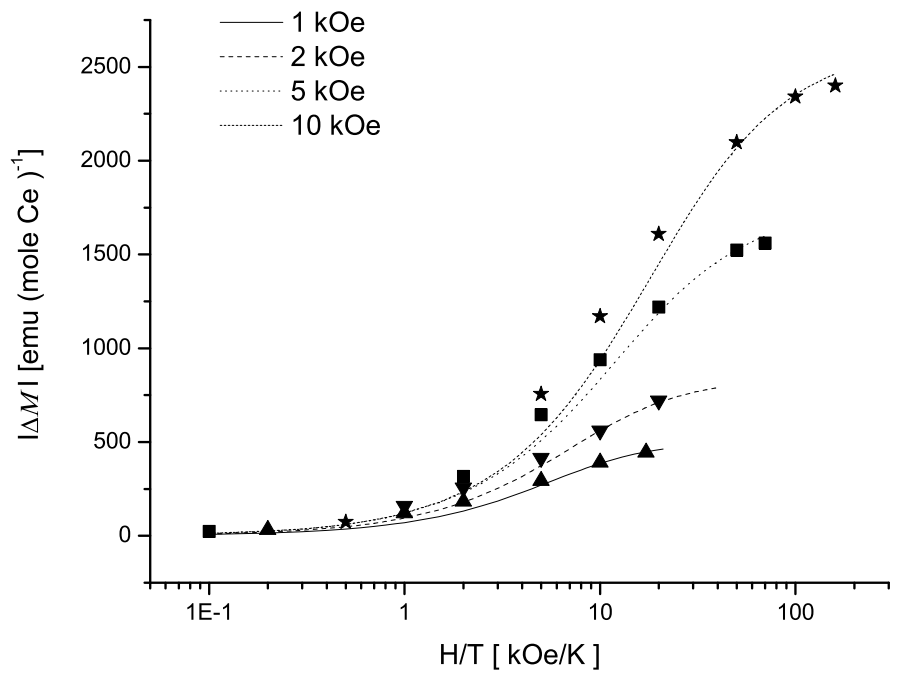

FIG. 9: The impurity magnetization $|\Delta M|$ of $\left(\mathrm{La}_{1-x} \mathrm{Ce}_{x}\right) \mathrm{Al}_{2}$ in various external magnetic fields as given by Eq. (32), with $x=$ 0.015 and $b_{0}, b_{1}, \gamma, M, \delta, \Delta T, g_{0}$ equal to the values in Table III The points are experimental results from Ref. 14 .

\section{MAGNETIC SUSCEPTIBILITY}

The zero-field impurity susceptibility

$$
\Delta \chi=\left(\frac{\partial \Delta M}{\partial \tilde{H}}+\frac{\partial \Delta M}{\partial \xi} \frac{\partial \xi}{\partial \tilde{H}}\right)_{\tilde{H}=0}
$$

has been the most frequently measured property of DMA. The theory of $\Delta \chi$, developed by Souletie et al $\stackrel{15}{\sim}$ for a DMA with RKKY interaction between impurities, predicts a dependence of the form

$$
\Delta \chi(T, c)=f(T / c)
$$

where $f$ is a function independent of concentration. Felsch et al $\stackrel{14}{\underline{4}}$ have confirmed approximate validity of Eq. (34) for $\left(\mathrm{La}_{1-x} \mathrm{Ce}_{x}\right) \mathrm{Al}_{2}$ with $x$ ranging from 0.02 to 0.06 .

Here validity of formula (33), with $\Delta M$ given by Eq. (32), is tested on $\Delta \chi$ experimental data for CuFe with $c=$ 110 ppm (Ref. 23) and (La1-x $\left.\mathrm{Ce}_{x}\right) \mathrm{Al}_{2}$ with $x=0.015,0.02$ (Ref. 14).

\section{A. $\mathrm{CuFe}$}

Daybell et al $\stackrel{23}{\underline{2}}$ expressed their measured $\Delta \chi$ values for CuFe in emu per gram of alloy per ppm. Formula (32), expressed in these units, takes the form

$$
\Delta M_{\mathrm{CuFe}}=\frac{1}{220} g^{\prime} \mu_{B} M_{\mathrm{Fe}} \tanh \left(t(\delta, T+\Delta T)\left(\frac{1}{2} g^{\prime} \mu_{B} \tilde{H}-\gamma \sqrt{n_{1}} \xi\right)\right)
$$

where $M_{\mathrm{Fe}}=1.042032405 \times 10^{18}$ is the number of Fe ions contained in one gram of $\mathrm{CuFe}$ with $c=110 \mathrm{ppm}$. A possible fit of the resulting function $\Delta \chi$, expressed in these units, to the experimental data on $\Delta \chi$, for CuFe from Ref. 23 is presented in Fig. 10. The corresponding values of the parameters are given in Table IV] Concavity of the $\Delta \chi\left(T^{-1}\right)$ curve in Fig. 10 appears not to be fully adjustable to concavity of the experimental plot at higher temperatures, however, agreement is satisfactory in the range of low temperatures. The discrepancy between theory and experiment at higher $T$ is presumably due to the increase of Fe spin values with increasing $T$ (Ref. 23). 


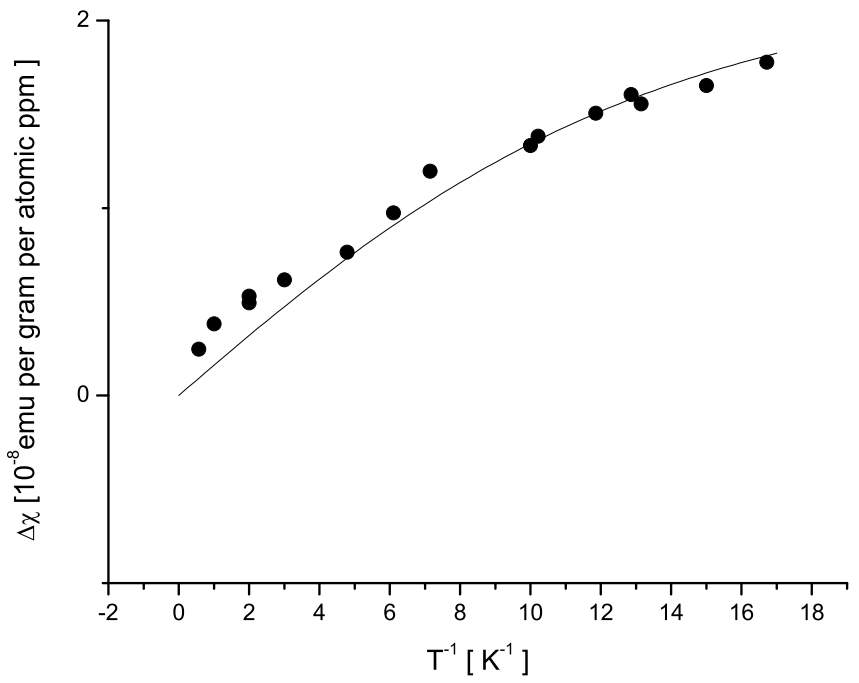

FIG. 10: The impurity susceptibility $\Delta \chi\left(T^{-1}\right)$ of CuFe, with $c=110 \mathrm{ppm}$, as given by Eq. (33), with $b_{0}, b_{1}, \gamma, M, \delta, g^{\prime}$, $\Delta T$ equal to the values in Table IV] The points are experimental results from Ref. 23 .

TABLE IV:

\begin{tabular}{|c|c|c|c|c|c|c|c|c|c|}
\hline Alloy & $x$ & $n_{1}$ & $b_{0}\left[10^{-10} \sqrt{\mathrm{erg}}\right]$ & $b_{1}$ & $\gamma\left[10^{-15} \sqrt{\mathrm{erg}}\right]$ & $M$ & $\delta\left[10^{-17} \mathrm{erg}\right]$ & $g^{\prime}$ & $\Delta T[\mathrm{~K}]$ \\
\hline $\mathrm{CuFe}$ & - & $10^{5} / 11$ & 8 & -101 & 1 & $10^{4}$ & 1 & 1.05 & 0.005 \\
\hline$\left(\mathrm{La}_{1-x} \mathrm{Ce}_{x}\right) \mathrm{Al}_{2}$ & 0.015 & $1592 / 3$ & 2.4048 & -13101 & 392370 & 200 & 1 & $10 / 7$ & 0.2 \\
\hline$\left(\mathrm{La}_{1-x} \mathrm{Ce}_{x}\right) \mathrm{Al}_{2}$ & 0.02 & $1192 / 3$ & 2.4048 & -13101 & 392370 & 400 & 0.1 & $10 / 7$ & 0.6 \\
\hline
\end{tabular}

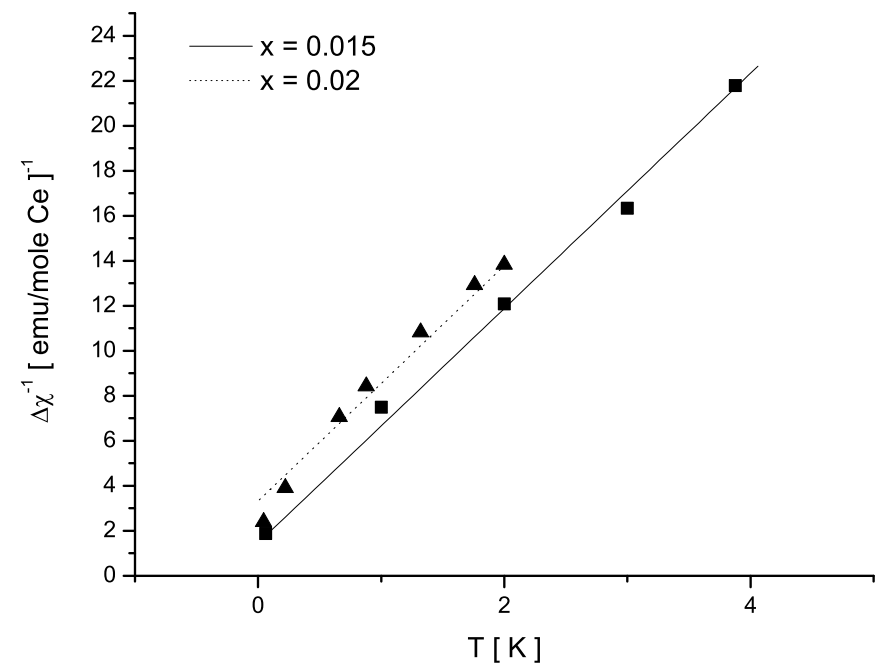

FIG. 11: The inverse impurity susceptibility $\Delta \chi(T+\Delta T)^{-1}$ of $\left(\mathrm{La}_{1-x} \mathrm{Ce}_{x}\right) \mathrm{Al}_{2}$ according to Eq. (33), with $x, b_{0}, b_{1}, \gamma, M, \delta$, $g^{\prime}, \Delta T$ equal to the values in Table IV] The points are experimental results from Ref. 14 . 


\section{B. $(\mathrm{LaCe}) \mathrm{Al}_{2}$}

Felsch et al ${ }^{14}$ performed detailed measurements of $\left(\mathrm{La}_{1-x} \mathrm{Ce}_{x}\right) \mathrm{Al}_{2}$ susceptibility for $x$ ranging from 0.01 to 0.2 . Their plots of $\Delta \chi(T)^{-1}$ for $x=0.01,0.015$ are almost indistinguishable and follow a Curie-Weiss law

$$
\Delta \chi(T)=\chi_{C F}(T) T /(T+\Theta), \quad \chi_{C F} T=\text { const }
$$

for $T \in(0.15 \mathrm{~K}, 3 \mathrm{~K})$. Below $0.15 \mathrm{~K}$ their data on $\Delta \chi(T)^{-1}$ for $x=0.015$, deviate from Eq. (36) to lower values, contrary to measurements of $\Delta \chi$ on $\mathrm{AuV}^{34}$ and earlier theories $4,20,35,36$ which predict a flattening-off of $\Delta \chi(T)^{-1}$ to higher values. For $x \geq 0.02$ the $\Delta \chi(T)^{-1}$ curves of Ref. 14 no longer obey Eq. (36) and exhibit a weak concavity.

Eq. (33), with $\Delta M$ expressed by Eq. (32), provides a good fit to the $\Delta \chi(T+\Delta T)^{-1}$ data of Ref. 14 for $x=0.01$, 0.015 and, as could be expected from Eq. (3), a less satisfactory adjustment for $x \geq 0.02$. The plots of $\Delta \chi(T+\Delta T)^{-1}$, as given by Eq. (33), are depicted in Fig. 11 for $x=0.015$, 0.02. A minor deviation from the Curie-Weiss law at very low temperatures, similar to the one found for $\mathrm{AuV}$ in Ref. 34, can be observed. The corresponding values of $b_{0}, b_{1}$, $\gamma, M, \delta$ are given in Table IV.

\section{CONCLUDING REMARKS}

The mean-field theory of dilute s-d systems presented in Ref. 16 has, in general, proved successful in providing quantitative explanation of the $T, c, H$ dependence of DMA heat capacity, magnetization and susceptibility in the range of low temperatures.

It has been shown that nonlinear dependence of DMA impurity heat capacity on $c$ can be explained in the dilute limit exclusively in terms of the s-d interaction, without introducing an impurity-impurity potential.

The EIT $t$ has improved the dependence of all thermodynamic functions on temperature and removed the singularity in Kondo's expression for DMA impurity resistivity.

Deviations of the theory from experiment, in the case of heat capacity, magnetization and CuFe susceptibility, are presumably due to increasing spin values of impurity ions observed in some DMA at higher temperatures and simplicity of the s-d coupling assumed. It has been suggested that, apart from s-wave, the coupling should account for the d-type character of the interaction.

Further improvement of the theory can be expected after including higher expansion terms of $f_{1}$ and correction terms to the mean-field free energy. The variation of impurity spin values at higher temperatures also suggests investigation of the thermodynamics of a more general s-d system containing impurity spins with various $S=1 / 2,1,3 / 2, \ldots$.

\section{APPENDIX}

To prove uniqueness of the minimizing solutions of the equations

$$
\begin{aligned}
& \frac{\partial}{\partial \xi} f\left(h^{(n, M)}(\xi, \eta), \beta\right)=0, \\
& \frac{\partial}{\partial \eta} f\left(h^{(n, M)}(\xi, \eta), \beta\right)=0,
\end{aligned}
$$

it suffices to note that Eqs. (A.1), (A.2) take the form (cf. Ref. 16)

$$
\begin{gathered}
\xi=f_{1}(\xi-\eta)+f_{2}(\xi), \\
\eta=f_{1}(\xi-\eta) .
\end{gathered}
$$

Thus, $\eta(\xi)=\xi-f_{2}(\xi)$, and therefore, for $\eta$ satisfying these equations 16 ,

$$
\frac{\mathrm{d}}{\mathrm{d} \xi} f\left(h^{(n, M)}(\xi, \eta(\xi)), \beta\right)=\frac{\partial}{\partial \xi} f\left(h^{(n, M)}(\xi, \eta(\xi)), \beta\right)=M\left(\xi-f_{1}\left(f_{2}(\xi)\right)-f_{2}(\xi)\right) .
$$

Since $f_{1}(x)=b_{0}+b_{1} x$ and $b_{1}<0,1 \ll\left|b_{1}\right|, f_{2}^{\prime}(\xi)>0$, it follows that the solution $\xi_{m}$ of Eq. (8) is unique and

$$
\frac{\mathrm{d}}{\mathrm{d} \xi} f\left(h^{(n, M)}(\xi, \eta(\xi)), \beta\right)<0 \quad \text { for } \xi<\xi_{m},
$$




$$
\frac{\mathrm{d}}{\mathrm{d} \xi} f\left(h^{(n, M)}(\xi, \eta(\xi)), \beta\right)>0 \quad \text { for } \xi>\xi_{m} .
$$

* ferm92@fizyka.umk.pl

1 J. Kondo, Prog. Theor. Phys. 32, 37 (1964).

2 P. E. Bloomfield and D. R. Hamann, Phys. Rev. 164, 856 (1967).

${ }^{3}$ K. Hepp, Solid State Commun. 8, 2087 (1970).

${ }^{4}$ K. G. Wilson, Rev. Mod. Phys. 47, 773 (1975).

5 V. T. Rajan, J. H. Lowenstein, and N. Andrei, Phys. Rev. Lett. 49, 497 (1982).

${ }_{7}^{6}$ N. Andrei, K. Furuya, and J. H. Lowenstein, Rev. Mod. Phys. 55, 331 (1983).

7 V. M. Filyov and P. B. Wiegmann, Phys. Lett. A 76, 283 (1980).

8 P. B. Wiegmann, in Quantum Theory of Solids, edited by I. M. Lifshits (MIR Publishers, Moscow, 1982).

9 A. C. Hewson, The Kondo Problem to Heavy Fermions (Cambridge University Press, Cambridge, 1993).

${ }^{10}$ K. Yosida, Theory of Magnetism (Springer, Berlin, Heidelberg, New York, 1998).

11 Y. K. Rudavskii, G. V. Ponedilok, and L. A. Dorosh, Cond. Matter Phys. 8, 579 (2005).

12 T. Kasuya, Prog. Theor. Phys. 16, 45 (1956).

13 J. P. Franck, F. D. Manchester, and D. L. Martin, Proc. Roy. Soc. (London) A 263, 494 (1961).

${ }^{14}$ W. Felsch, K. Winzer, and G. V. Minnigerode, Z. Physik B 21, 151 (1975).

15 J. Souletie and R. Tournier, J. Low Temp. Phys. 1, 95 (1969).

16 J. Maćkowiak, Physica A 336, 461 (2004).

17 J. Maćkowiak (2007), to be published, Preprint arXiv:cond-mat/0703146.

18 B. Simon, Functional Integration and Quantum Physics (Academic Press, New York, San Francisco, London, 1979).

19 H. Kummer, J. Math. Phys. 8, 2063 (1967).

${ }^{20}$ P. W. Anderson, G. Yuval, and D. R. Hamann, Phys. Rev. B 1, 4464 (1970).

21 S. F. Edwards, Phil. Mag. 3, 1020 (1958).

22 V. Ambegaokar, in Superconductivity, edited by R. D. Parks (Marcel Dekker, Inc., New York, 1969).

23 M. D. Daybell and W. Steyert, Phys. Rev. Lett 18, 398 (1967).

24 A. J. Heeger, in Solid State Physics, edited by F. Seitz, D. Turnbull, and H. Ehenreich (Academic Press, New York, 1969), vol. 23

25 D. R. Hamann, Phys. Rev. 158, 570 (1967).

${ }^{26}$ G. J. van den Berg, Prog. Low Temp. Phys. 4, 194 (1964).

27 D. C. Mattis, The Theory of Magnetism I (Springer, Berlin, Heidelberg, New York, 1988).

28 S. D. Bader, N. E. Philips, M. B. Maple, and C. A. Luengo, Solid State Commun. 16, 1263 (1975).

29 B. B. Triplett and N. E. Philips, Phys. Rev. Lett. 27, 1001 (1971).

30 C. F. Brock, J. C. Ho, G. P. Schwartz, and M. E. Philips, Solid State Commun. 8, 1139 (1970).

31 A. Aharoni, Introduction to the Theory of Ferromagnetism (Oxford University Press, 2000).

32 P. Monod and S. Schultz, Phys. Rev. 173, 645 (1968).

33 J. R. Schrieffer, J. Appl. Phys. 38, 1143 (1967).

34 J. E. van Dam, P. C. M. Gubbens, and G. J. van den Berg, Physica 61, 389 (1972).

35 P. W. Anderson and G. Yuval, Phys. Rev. Lett. 23, 89 (1969).

${ }^{36}$ K. D. Schotte and U. Schotte, Phys. Rev. B 4, 2228 (1971). 